\title{
Simulation of Flow Field Past Symmetrical Aerofoil Baffles Using Computational Fluid Dynamics Method
}

\author{
Tarek J. Jamaleddine, Ramsey Bunama \\ SABIC Plastics Application Development Center \\ Riyadh Techno Valley, Riyadh, KSA \\ Jamaleddine@sabic.com; Bunamar@sabic.com
}

\begin{abstract}
Compressors are widely used in the petrochemical industry to provide a variety of gas properties that are essential for a variety of chemical processes. Among others, ambient air is predominantly used due to its abundancy in nature and its richness in oxygen. It is customary that clean air is supplied to the compressor by means of a commercial air intake filter placed few meters upstream of the compressor inlet. Various ducting configurations are commercially utilized to link the air filter to the compressor inlet. Embedded baffle-type muzzling units are placed inside the ducting for attenuating acoustic noise generated by the incoming high speed compressible flow. It is imperative that these silencer baffles are aerodynamically structured to maintain constant flow conditions dowsntream of the trailing edge for sustaining an efficient compressor operation. In the Gulf region where ambient temperature condition during hot summer day surpasses $45^{\circ} \mathrm{C}$ coupled with saturated moist air, controling and maintaining constant conditions upstream of the compressor inlet pose a great challenge. In this paper, we introduce a numerical study employing Computational Fluid Dynamics (CFD) method for predicting the hydrodynamic conditions within the geometrical entity linking the air filter to the compressor inlet boundary in the presence of a set of symmetrical and cusped trailing-edge aerofoil-shaped silencers. Several baffle locations were investigated to analyze the impact of different locations on the pressure losses and temperature conditions upstream of the compressor inlet. Results show that an optimized silencer location can stabilize the flow conditions upstream of the compressor inlet. Among the considered locations, a recommendation was given for the best location that led to a reduced pressure drop across the bluff body and stabilized inlet conditions to the compressor.
\end{abstract}

Keywords: CFD, compressors, aerodynamic flows, acoustic baffles, silencers, pressure loss

\section{Introduction}

Gases and chemicals are vital resources for the petrochemical industry. Some of which are freely available and easily merged into the main process stream, while others require pre-processing prior to their use in the chemical process. Among those readily available and easily employed, air is one of the most vital element used in the process stream due to its richness in oxygen and nitrogen.

Some of the well-known chemical technologies utilize air to extract oxygen for processes involving combustion, others co-feed it with the inlet stream. Traditionally, clean air is supplied to the plant by means of an industrial scale intake air filter that sieves the air from undesirable fines prior to directing it to an in-line turbine compressor. In the compressor, air properties such as pressure and temperature are thermodynamically increased to the desired process condition. Since the compressor efficiency is a direct function of the air characteristics at the compressor inlet, it is crucial that constant inlet conditions are maintained throughout the operation cycle. Any changes in the upstream inlet conditions could result in an increase in power consumption of the compressor, or in some extreme cases, unforeseen problem with compressor functionality.

Inlet conditions of temperature and pressure are sensitive to various factors, the most common are fluctuations in environmental conditions. When silencers are also installed in the ducting system, their presence adds another degree of complexity to the flow field and therefore to the design of filters. These units are commonly aerodynamically structured to have minor impact on the flow field downstream of the trailing edge and on the pressure losses across their surfaces. Lewis [1] shed light on the impact of various aerofoil trailing edge geometries on the vorticity shedding in the wake region. Among the three studied trailing edge geometries namely, cusp, round, and sharp, flow field over the cusped trailing edge showed equal and opposite quantities of vortices shed from the upper and lower surfaces. This phenomenon resulted in 
cancellation of vortices and subsequent stabilization of the flow downstream of the trailing edge. Rhie et al. [2] cautioned against the use of standard $k-\epsilon$ turbulence model in flows with separation as it predicted fairly good pressure distribution over NACA 0012 airfoil surface compared to attached flows, where the model predicted reasonably good agreement with the experimental data. Khan et al. [3] employed CFD method to recommend the most effective location for installing a fogging system with respect to the location of the silencer baffles in improving the cooling and evaporative effectiveness during hot summer days. Additional factor that has contributed to the evaporative effectiveness and to the pressure drop is the number and randomness of baffles placed in the flow field. The authors pointed out that staggered silencers augmented the pressure drop by $6.5 \%$ than a single row of silencers.

In this paper, we present a 3-D numerical study employing CFD method for predicting the hydrodynamic conditions within a duct system linking an air intake filter to a compressor inlet boundary in the presence of a set of symmetrical and cusped trailing-edge aerofoil-shaped silencers. The objective of the CFD simulation is to provide a quantitative information of the pressure losses in the presence of silencers, and subsequently recommend an optimum location for placing the baffles that could potentially lessen the pressure drop in the system. The k- $\omega$ turbulence model with the Shear-Stress Transport (SST) advantage was adopted with medium-to-fine size mesh in the overall computational domain, except in the proximity of the domain wall and the leading and trailing edge regions where dense mesh was imposed to accurately capture the pressure distribution and substantially improve the accuracy of the downstream results. CFD predictions showed that baffles location has a profound effect on the flow field upstream of the compressor inlet.

\section{Model Description}

\subsection{CAD Model Development}

A 3D CAD model of the considered geometry is shown in Figure 1. The computational domain measures $10.4 \mathrm{~m}$ in length with an outlet cross sectional area of $2.54 \mathrm{~m} 2$ and an inlet-to-outlet area ratio of 18 . During normal operation, ambient air at $21.0 \mathrm{oC}$ is drawn into an industrial scale air filter for screening before entering the transition duct. It then travels past a set of symmetrical aerofoil baffles, also known as industrial silencers for this particular application, to a circular duct before entering the compressor. The primary purpose of the industrial silencers is to attenuate acoustic noise generated by the high speed incoming flow. These silencers however have the tendency to disturb the flow field and augment pressure losses. It is therefore critical that their impact on the flow field is within a desirable range to achieve an optimum and sustainable upstream condition for the compressor inlet.
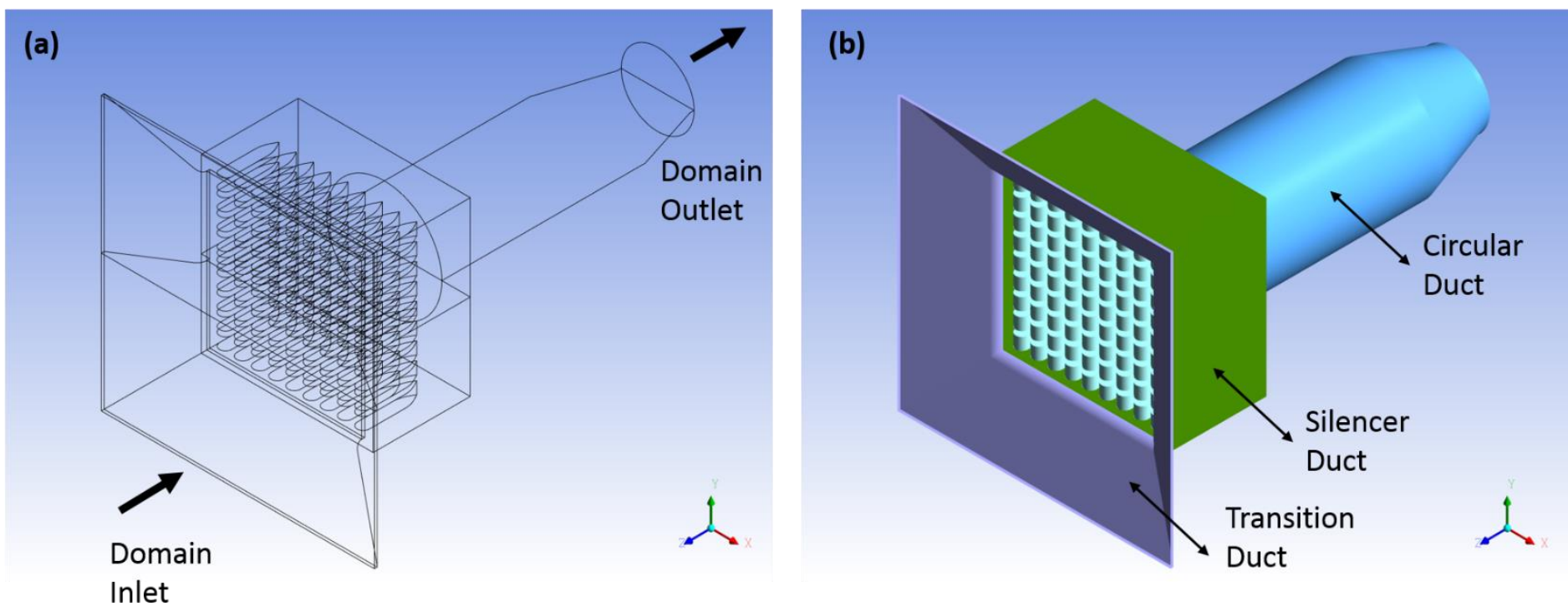

Fig. 1: Model geometry, (a) 3D schematic drawing showing inlet and outlet domains, (b) 3D CAD model identifying different features in the computational domain.

Two cases were studied based on the location of the silencers in the longitudinal z-direction (Figure 2). These cases depict the customary silencer locations adopted in the industry. The first case marks the silencers leading edge at the inlet 
of the silencer duct downstream of the transitional duct. The second case however specifies an offsetted leading edge location by $756 \mathrm{~mm}$ from the initially considered case towards the circular duct inlet.

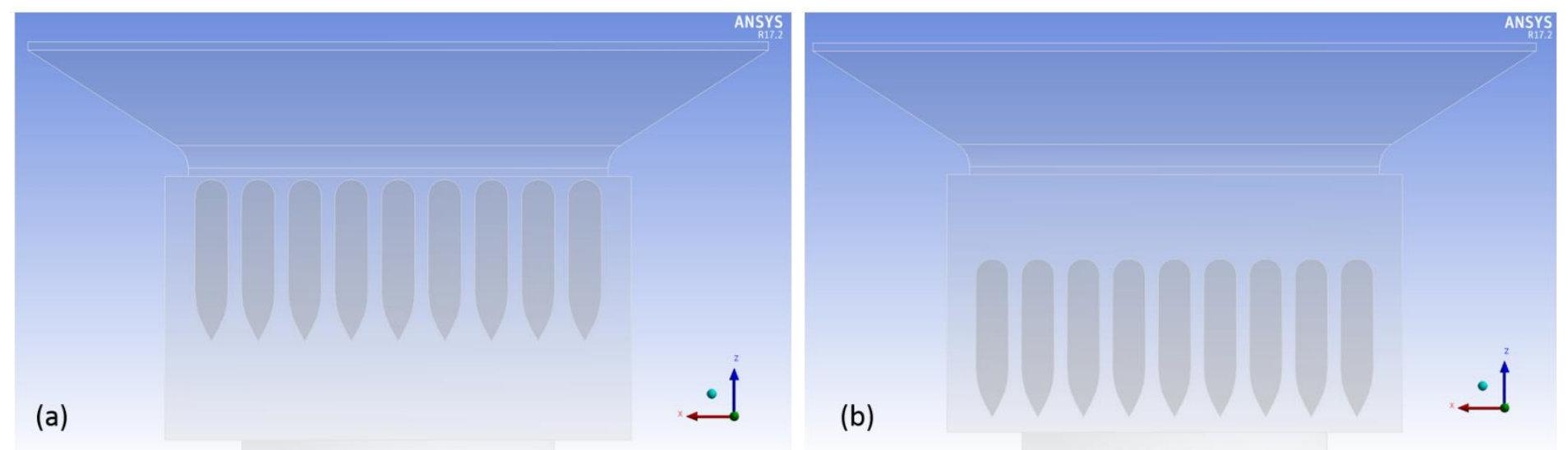

Fig. 2: Bottom view of silencer location along the symmetry plane for (a) Case 1 and (b) Case 2.

\subsection{CFD Model Development}

Initially, the model geometry was created in ANSYS DesignModeler. Meshing was then introduced using ANSYS Workbench meshing capability. The meshed model was then analyzed for the flow field and temperature distribution using ANSYS Fluent single phase flow solver. Due to geometrical symmetry, half portion of the model was simulated to conserve computational effort whilst still obtaining accurate prediction. The meshed model is shown in Figure 3.
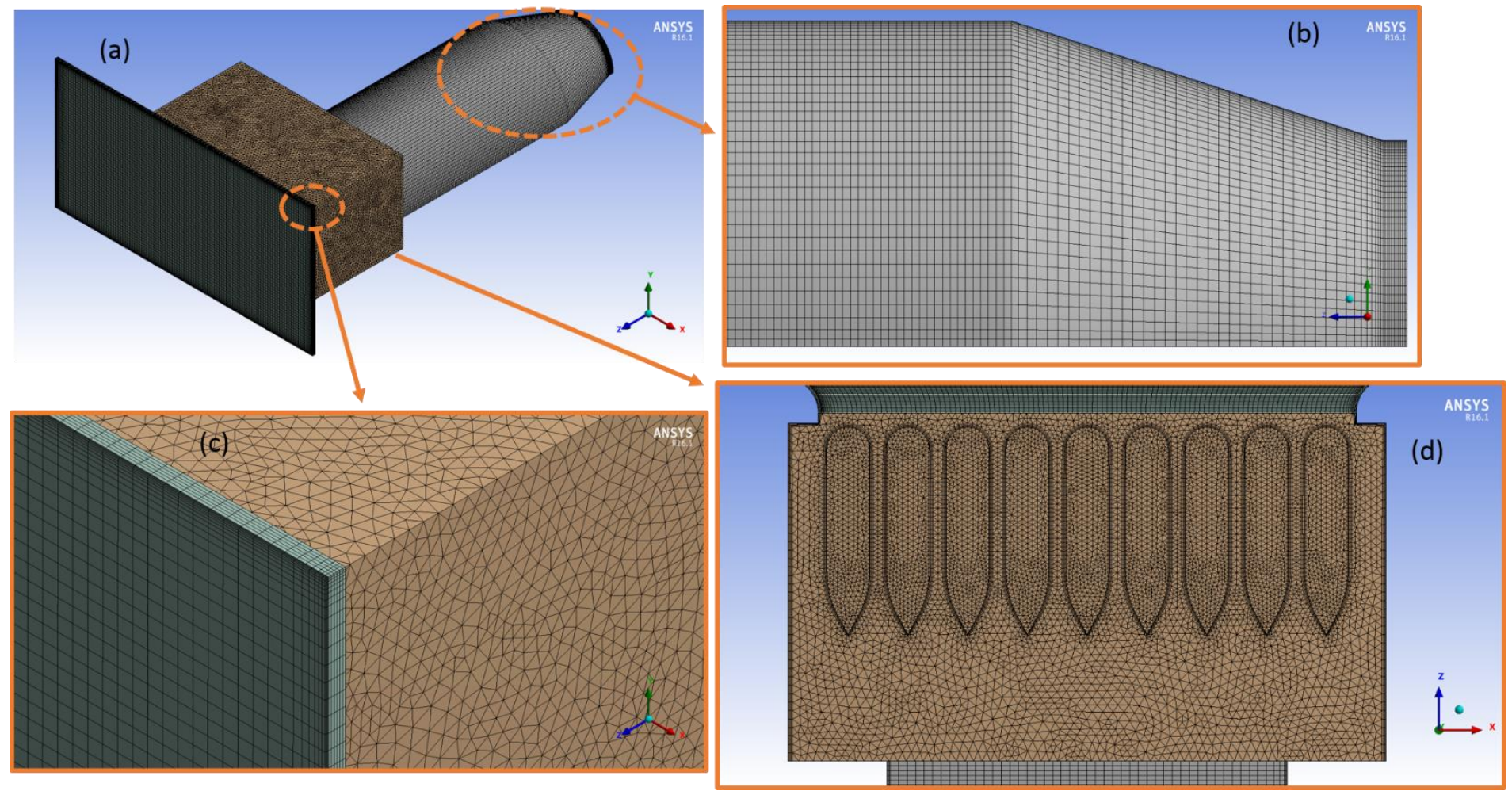

Fig. 3: Meshed model, (a) overall meshed model, (b) enlarged frontal view showing fine mesh near the wall and outlet region, (c) enlarged inlet domain, (d) bottom view of silencer region along the symmetry plane. 
Hexahedral type elements were predominantly applied in the circular section to accurately capture the flow properties. These elements provide superior quality over other meshes type when aligned in the direction of the flow. Due to geometrical complexity in the silencer and transitional regions, tetrahedral and mixed type elements were implemented. Finer mesh was implemented close to the domain walls to account for the viscous effect at the wall and to accurately resolve the gradients in the boundary layer region (Refer to Figure 3).

The governing equations were solved for a compressible gaseous flow using the pressure-based SIMPLE algorithm double-precision solver in ANSYS Fluent v17.2. The Shear-Stress Transport (SST) k- $\square$ turbulent model was adopted since it provides more accurate results for those class of flows involving adverse pressure gradients, airfoils, and transonic shock waves. It took 3 days of wall-clock time for a 0.05 -second transient solution to converge on an 8-Core 16-node Intel processor with residual mean values below 10-4 for flow and turbulent equations and 10-6 for energy.

At the inlet of the computational domain, an air temperature of $21.0 \mathrm{oC}$ and a mass flow rate of $74 \mathrm{~kg} / \mathrm{s}$ were specified. Gas properties were then derived based on the ideal gas law for compressible flow. At the domain outlet, a pressure outlet condition was specified. A no-slip boundary condition with an adiabatic wall temperature was specified at the domain walls, and a continuous boundary condition (zero normal gradients of all variables) was specified at the symmetry planes.

\subsection{Grid Sensitivity Study}

A grid independency study was performed on Case 1. The total element count and element type for both mesh sizes is shown in Table 1. For both cases, the global element quality was reported to be greater than 0.12 and the global cell skewness less than 0.89. This ensures the best setting for more accurate prediction and more guaranteed converged solution.

Table 1: Mesh count for Cases $1 \& 2$.

\begin{tabular}{|c|c|c|c|c|}
\hline & Mesh quality & Total elements & Hexahedral elements & Mixed elements \\
\hline \multirow{2}{*}{ Case 1} & Coarse & 5398117 & 336072 & 5062045 \\
\cline { 2 - 5 } & Finer & 7750621 & 2688576 & 5062045 \\
\hline Case 2 & Fine & 8433248 & 336072 & 8097176 \\
\hline
\end{tabular}

The predicted pressure drop for different mesh sizes is shown in Figure 4. In this figure, the $x$-axis spans the length of the computational domain from the inlet of the circular duct to the overall domain outlet, in the negative longitudinal $\mathrm{z}$ direction. It can be clearly seen in the figure that the coarse mesh grossly overestimated the pressure drop in that region compared to the predicted results with fine mesh. Consequently, fine mesh was adopted for Cases $1 \& 2$. 


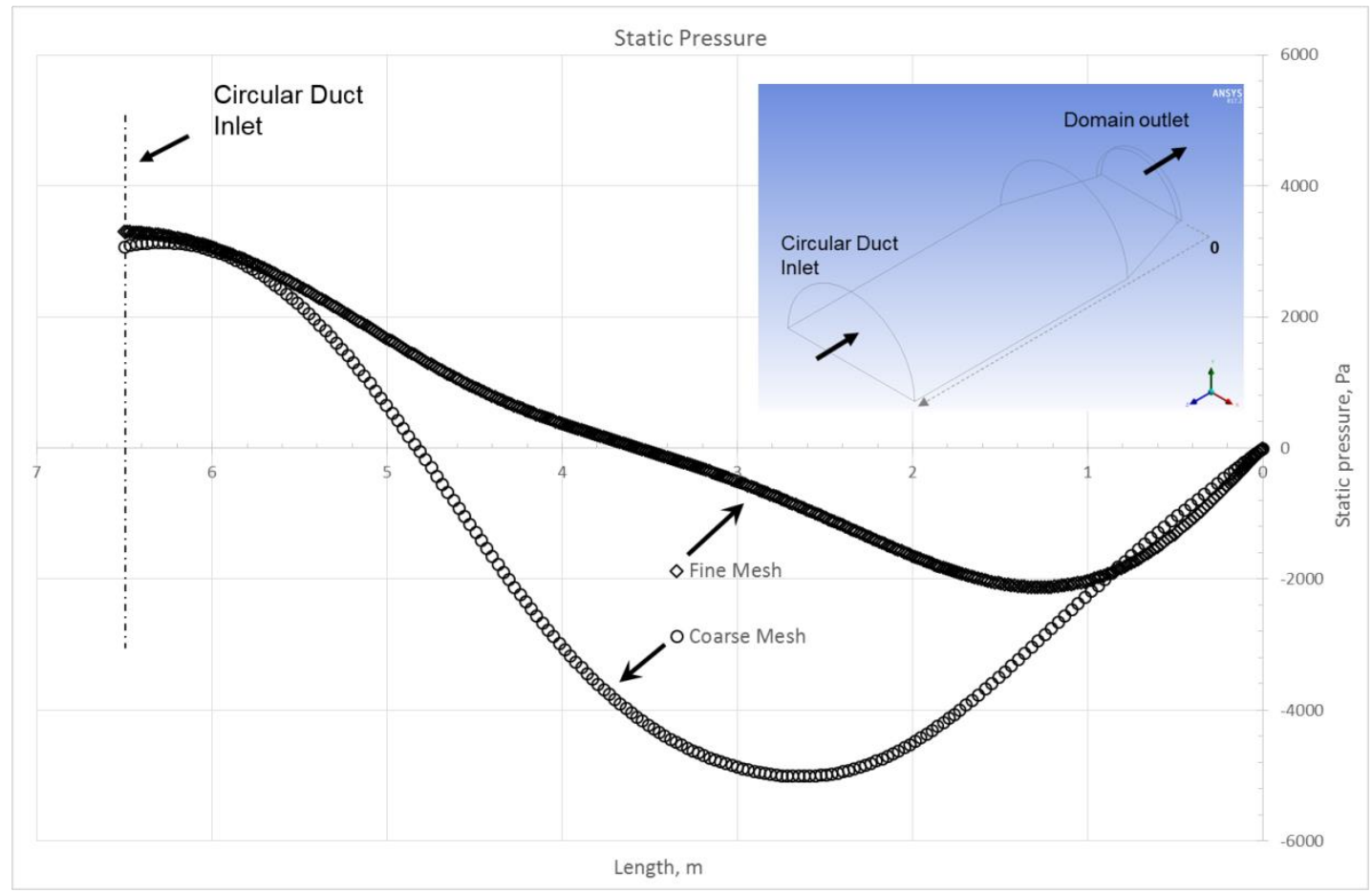

Fig. 4: Plot of static pressure for Case 1 across the circular duct.

\subsection{Convergence Criterion}

Several criteria were adopted to determine solution convergence. Most importantly, mass balance was conserved at the domain boundaries. The residual mean values for the conservation equations of mass, momentum, and turbulence were set to fall below $10^{-4}$ and for the energy below $10^{-6}$. Solution variables such as pressure, temperature, Reynold and Mach

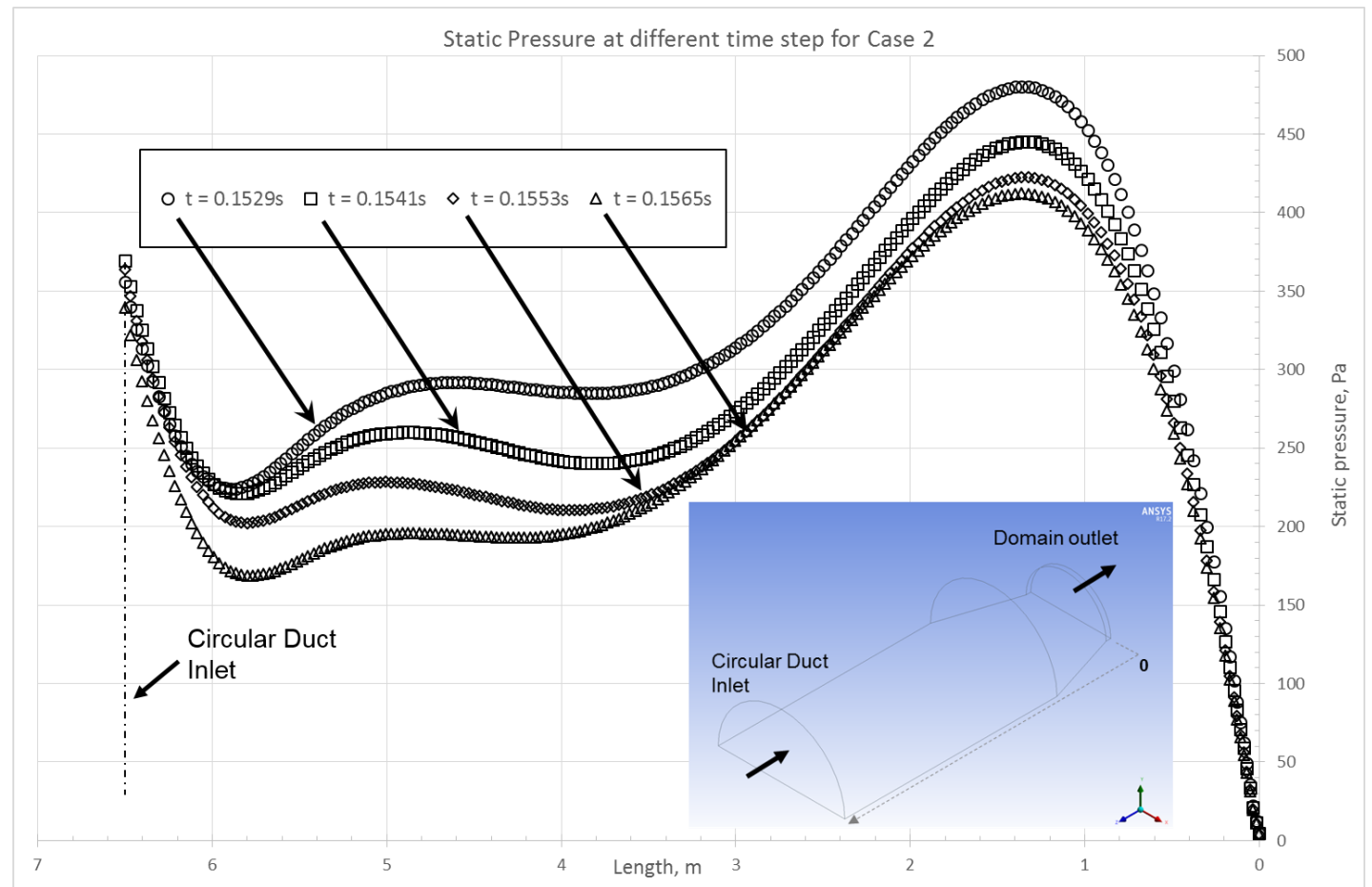

Fig. 5: Plot of static pressure for Case 2 across the circular duct. 


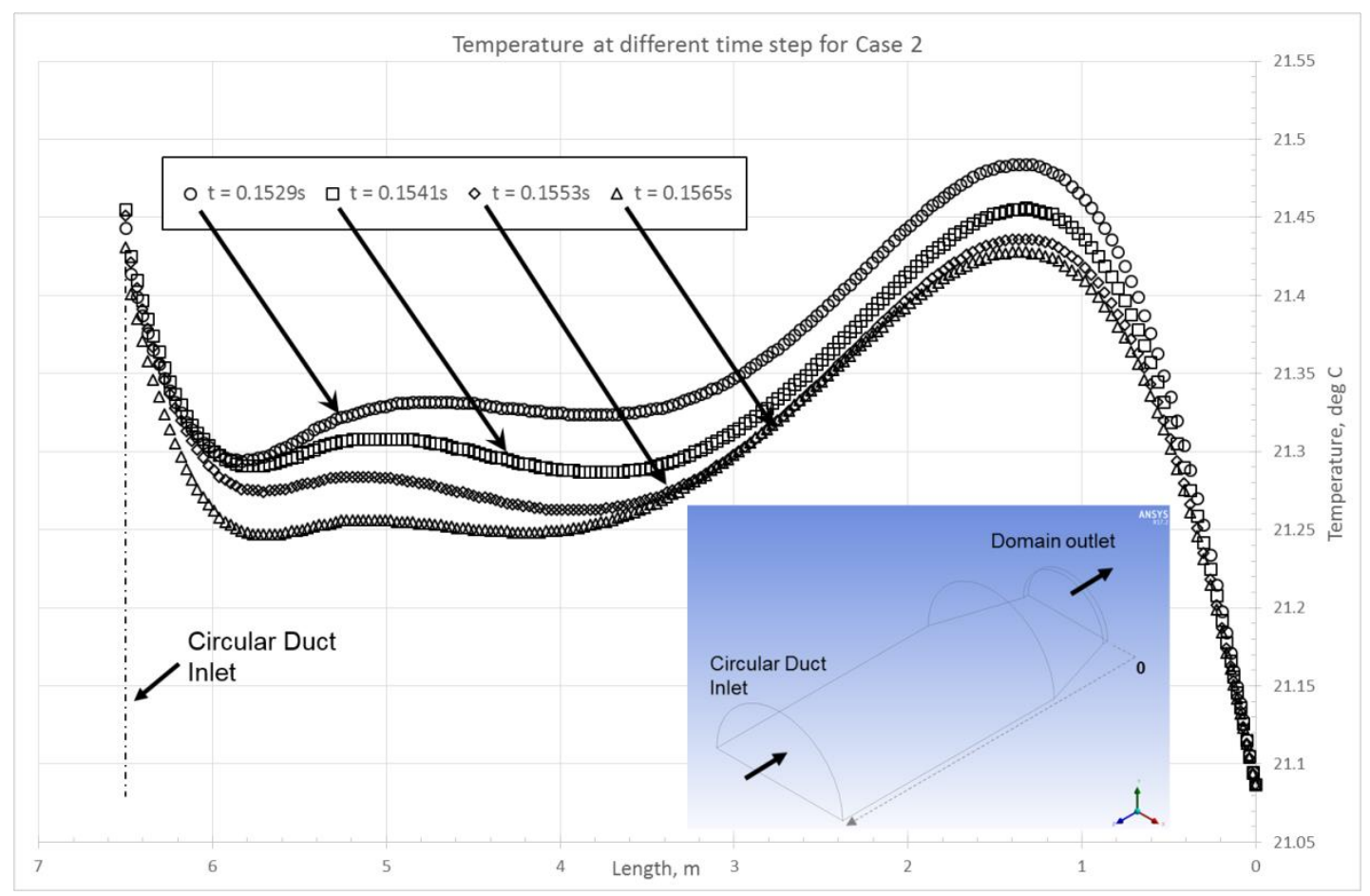

Fig. 6: Plot of air temperature for Case 2 across the circular duct.

numbers were all monitored during the iteration process as an additional measure of convergence. These variables were plotted for consecutive iterations across the circular duct and were examined for a smoothly converging solution. For illustration purposes, Case 2 predictions for pressure and temperature values are shown in Figures $5 \& 6$, respectively. It is clearly evident from both plots that a steady state solution was achieved from the transient simulation.

\section{Discussion of Results}

In the previous sections, pressure profiles were separately plotted for each considered case. These profiles were replotted in Figure 7 to easily capture the difference between both cases due to changes in silencers location within the silencer duct. It can be concluded from the predicted profiles that Case 2 exhibited smaller variation compared to Case 1 which showed much greater drop along the entire duct length from inlet to outlet. The predicted pressure for Case 1 at the duct inlet was nearly 900\% higher than Case 2 at the same location, and nearly $1500 \%$ lower close to the domain outlet. Case 2 showed less overall pressure drop with slight changes along the duct length. This confirms a more stable condition upstream of the compressor inlet. Similar phenomenon was also observed with the temperature distribution as shown in Figure 8; nevertheless, the differences between both cases were not as pronounced as for the pressure profiles. 


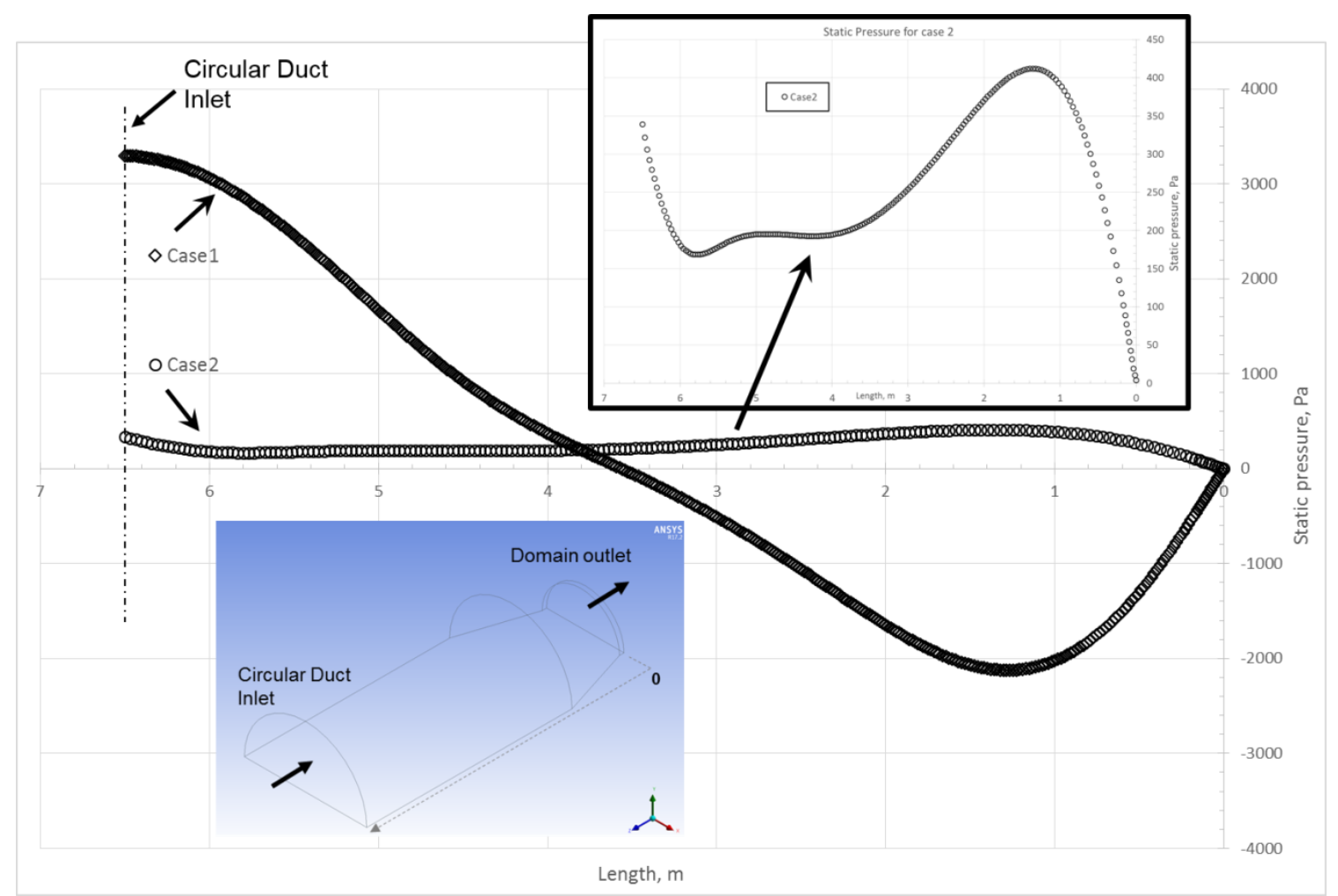

Fig. 7: Plot of static pressure for Cases $1 \& 2$ across the circular duct.

The presence of silencers in the fluid domain created a relatively complex hydrodynamic pattern downstream of the silencer duct for both cases. This complex behavior is clearly presented by Reynolds number profile in Figures 9 and 10 . Although both cases observed the three modes of flow regimes, namely, laminar, transitional, and turbulent, the extent and magnitude of each regime were distinct. For Case 1, the extent of turbulence was lesser while the transitional flow was more pronounced and prolonged towards the outlet of the circular duct. A region of chaotic separated flow occurred for a longer distance before a fully turbulent regime was observed. Higher pressure loses might have been attributed to this extended transitional state. This in turn would create a less stable flow condition entering the compressor inlet. For Case 2 however, the flow accelerated just upstream of the converging region and within while approaching the compressor inlet. The magnitude of turbulence behavior in the converging region marked a fast moving flow with smoothed-out velocity differences, and thus, low pressure losses. Upstream of that region, the flow encountered a very short transitional segment preceded by a predominantly laminar flow. 


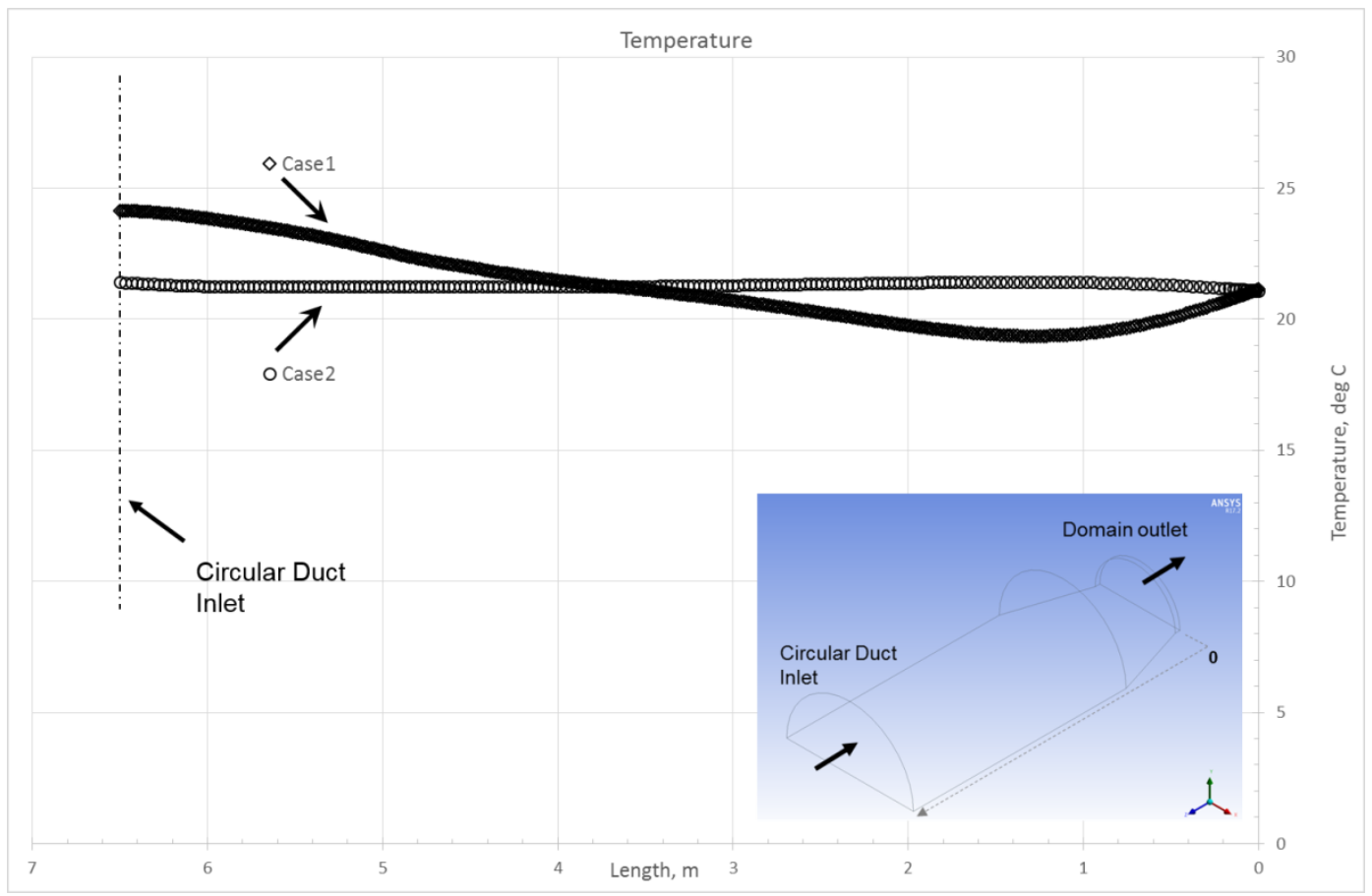

Fig. 8: Plot of temperature profile for Cases $1 \& 2$ across the circular duct.

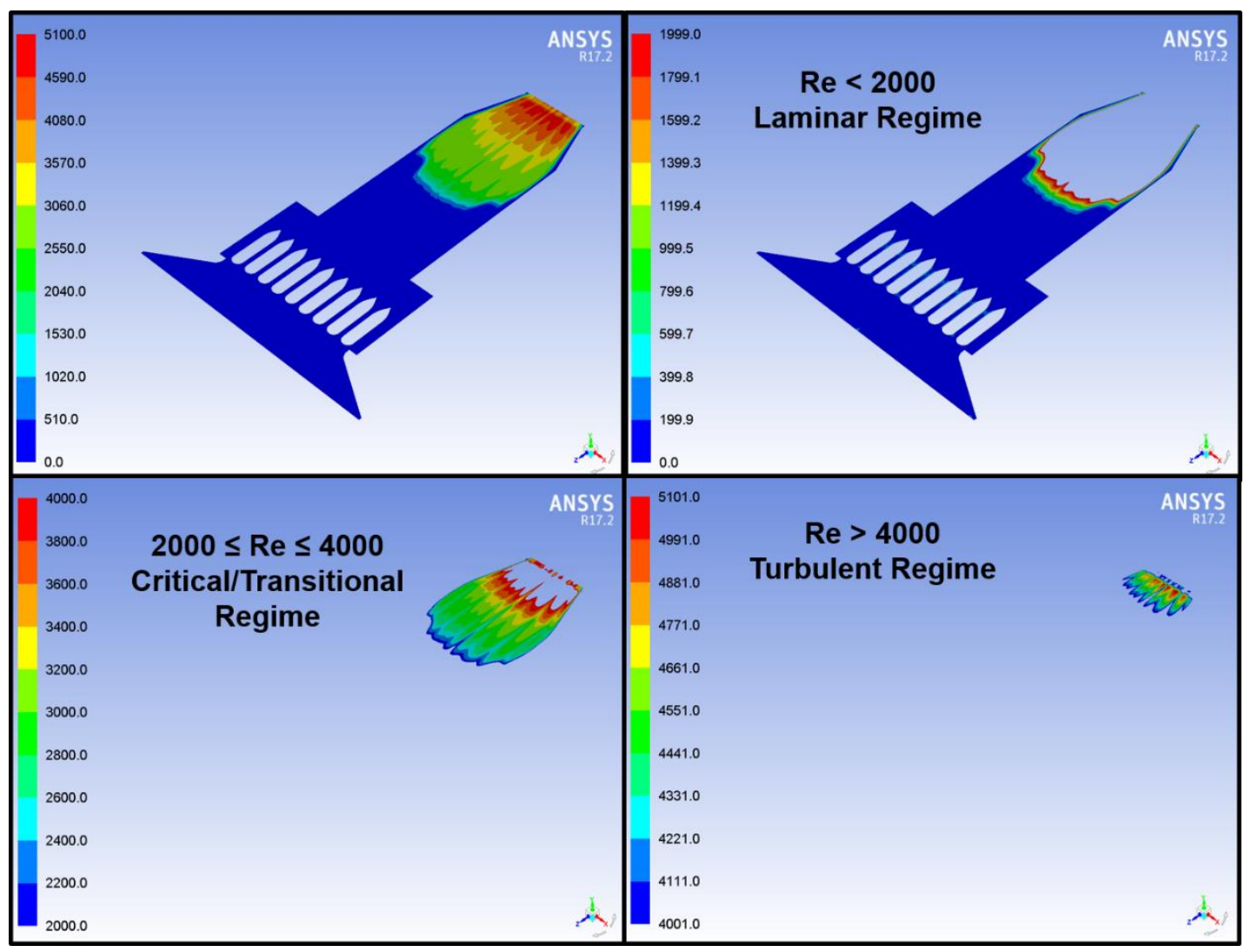

Fig. 9: Reynolds number for Case 1 for ALL flow regimes across the entire fluid domain. 


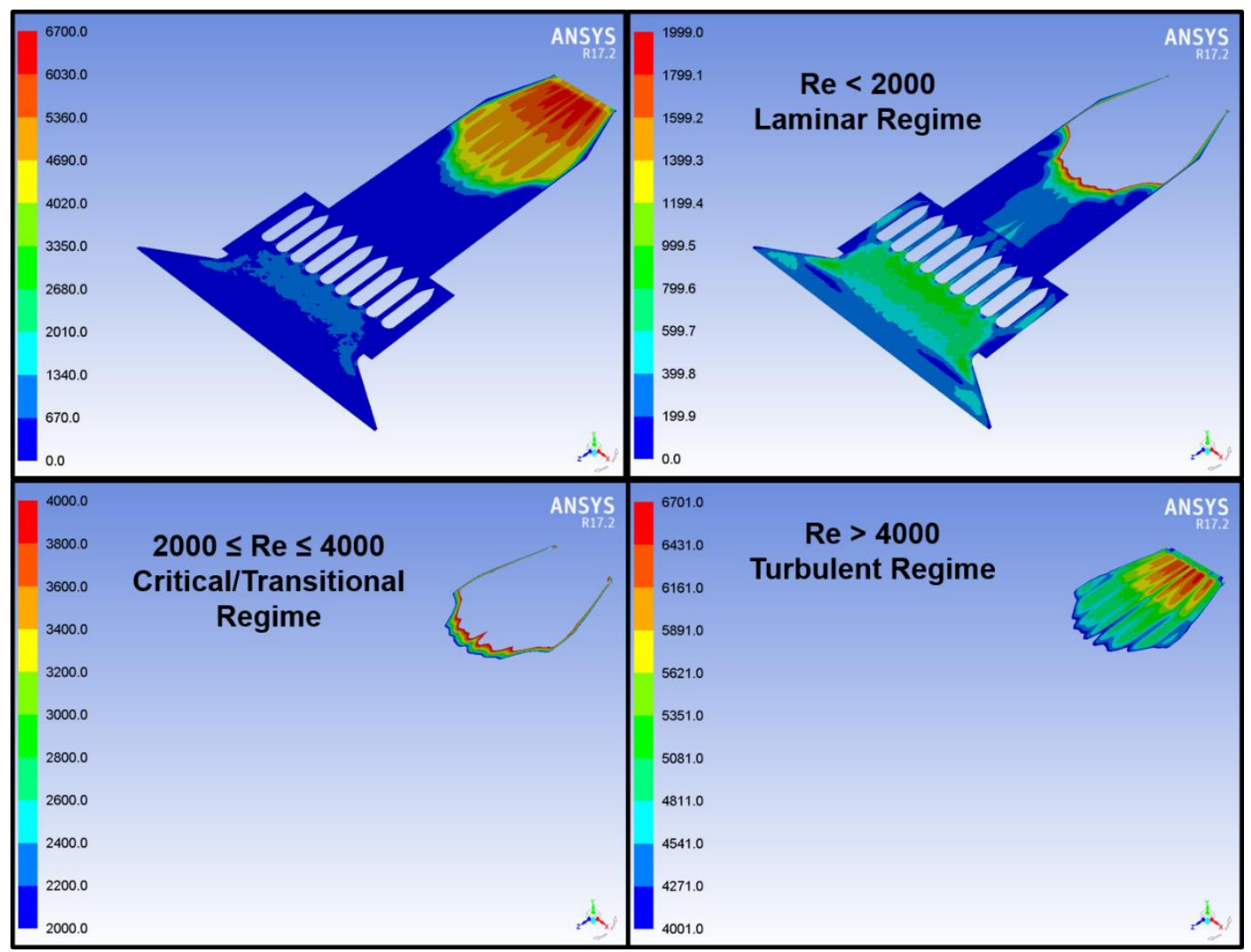

Fig. 10: Reynolds number for Case 2 for ALL flow regimes across the entire fluid domain.

Additional interesting observation from the above figures can be drawn. Not only that the silencers presence in the flow direction had a substantial impact on the pressure losses in the system, their location with respect to the silencer duct boundaries also played a crucial role on the flow characteristic and mixing process behind the trailing edge. In Case 2 for instance, by offsetting the leading edge away from the silencer duct inlet, nonuniform flow on cross sections perpendicular to the flow direction persisted for a moderately long distance along the length of the circular duct ahead of the transitional regime. Higher velocity profile was also observed in the centerline of the duct, which contributed to lower pressure drops as previously shown in Figure 7. Downstream of the transitional regime, flow streamlines converged just downstream of the diameter change accompanied with a decrease in pressure and temperature as shown in Figures $7 \& 8$.

The pressure profile across the baffles is depicted in Figure 11. Case 2 showed more intricate profile than Case 1. The pressure increased in both cases along the baffles length; nevertheless, for Case 2, this increase was only noticeable along the path for those baffle layers in the proximity of the silencer duct wall. This behavior was anticipated since the trailing edge of these baffle layers were close to the 90 degree sharp corner at the duct outlet. The predicted pressure values in these regions were the highest in compatible with regions of stagnation pressure. In Case 1 however, pressure gradually increased along the length of the baffles from the leading edge to the trailing edge with more uniform distribution along the silencers duct width.

The above discussion in regards to the pressure distribution in the silencer duct region brings an intriguing observation. Depending on the considered application, setting an industrial equipment just downstream of the silencer duct for Case 2 would not be prudent. The non-uniformity of pressure distribution exiting the duct could potentially lead to a weak and inefficient system performance. The equipment should be placed at far away distance downstream of the duct outlet boundary as in the case of the geometry shown in this study. On the contrary, placing an equipment few distances away from the silencer duct outlet could be advantageous in cases where the pressure distribution is similar to that in Case 1 , since this option would shorten the circular duct length while still attaining less pressure losses and achieving more stable and desired conditions into the compressor inlet. 


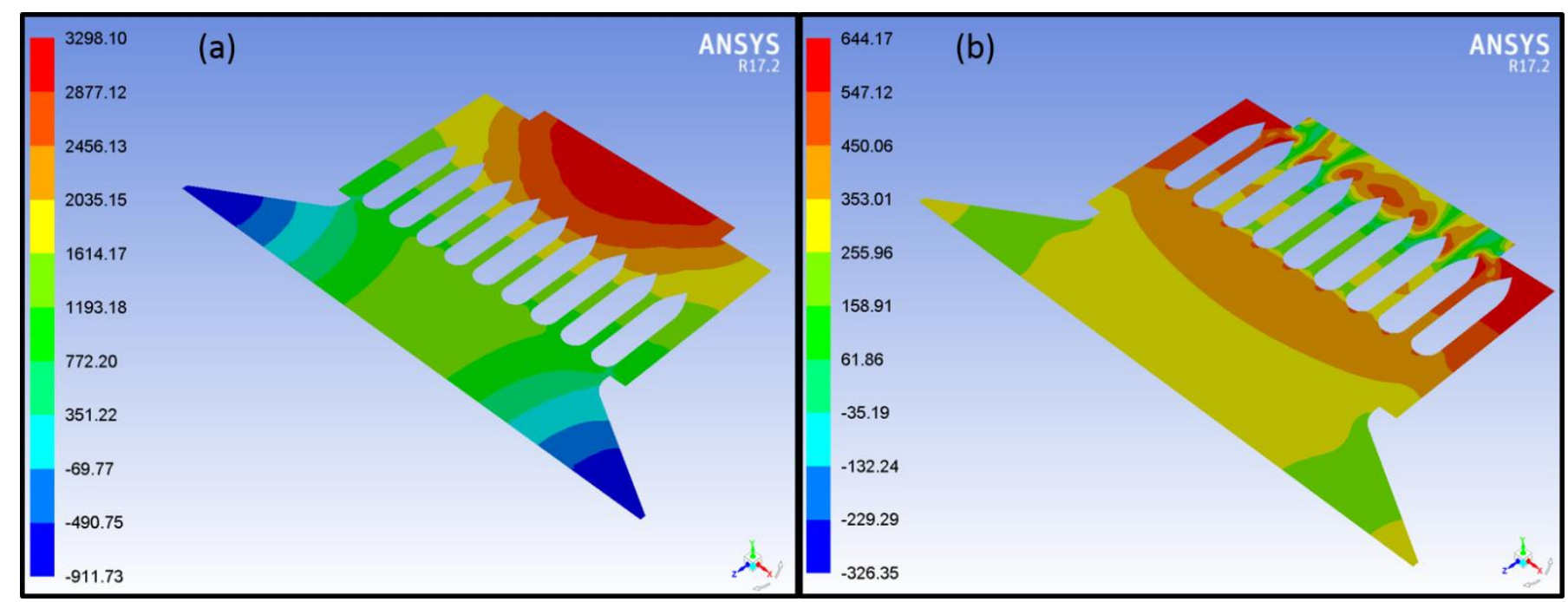

Fig. 11: Pressure profile for both cases across the baffles, (a) Case 1, (b) Case 2.

\section{Conclusion}

The simulation of the flow field of a ducting system linking an industrial scale air filter to an air compressor was carried out using CFD analyses. Two cases were analyzed based on the location of the silencers in the fluid domain. Both cases experienced different flow characteristics with respect to silencer locations. Numerical model predictions showed a complex flow pattern along the length of the circular duct leading to the compressor inlet. Both models experienced Reynolds numbers ranging from laminar to transitional to fully turbulent flow. For the case where the silencers leading edge was in the proximity of the duct inlet, a more pronounced and prolonged transitional flow nearly mid-way of the circular duct length was predicted followed by a short turbulent flow before entering the compressor inlet. A distinct behavior was predicted for the case where the silencers trailing edge was in the proximity of the duct outlet boundary. Pressure losses in the circular duct for the later case were also comparatively less.

At the silencer duct outlet, pressure predictions for Case 1 were higher than Case 2 and more uniformly distributed along the length of the silencers. A more chaotic and non-uniform distribution was observed for the silencer location of Case 2. Conclusively, for an industrial equipment to perform efficiently and to maintain a stable operating condition, it should be placed either at fewer distances downstream of the duct outlet for moderately uniform pressure distribution across the silencers length, or at far away distance from the duct outlet for non-uniform pressure distribution.

\section{References}

[1] R. I. Lewis, "Surface vorticity modelling of separated flows from two-dimensional bluff bodies of arbitrary shape," Journal Mechanical Engineering Science, vol. 23, no. 1, pp. 1-12, 1981.

[2] C. M. Rhie and W. L. Chow, "Numerical study of the turbulent flow past an airfoil with trailing edge separation," AIAA Journal, vol. 21, no. 11, pp. 1525-1532, 1983.

[3] J. R. Khan, T. Wang and M. Chaker, "Investigation of cooling effectiveness of gas turbine inlet fogging location relative to the silencer," in Proceedings of ASME Turbo Expo, Vancouver, Canada, pp. 1-12, 2011. 